The Egyptian Journal of Hospital Medicine (October 2019) Vol. 77 (3), Page 5235-5241

\title{
Mobility Safety in Chronic Elderly Stroke: Evaluation and Assessment A Narrative Review of the Current Literature and Future Directions
}

\author{
Bani Ahmed Ali A.
}

Department of Physical Therapy, Faculty of Applied Medical Sciences, University of Tabuk

Mobile: (00966) 533933971, email: ali.baniahmed@gmail.com

\begin{abstract}
Purpose: Safe ambulation is a multidisciplinary approach of combined skills of medical, nursing, and physical therapy staff to achieve safe outcomes within in- and outpatient settings. The purpose of this literature review is an in-depth evaluation of the available research on factors contribute to mobility falls in chronic ambulatory stroke survivors as a vascular brain pathology with senseromotor consequences especially in geriatric population. Most importantly, the review proposes guidelines for a comprehensive assessment tool combining the major threats assessed of mobility safety in integrated approach.

Material and Methods: This narrative review searched in PubMed/MEDLINE was presented in four sections: (Section one) represents the gait motor impairment, (section two) represents the gait motor recovery (section three) addresses walking speed and how it relate to community ambulation and (section four) represents the major threats to mobility safety in chronic stroke survivors.

Results: The three major factors that are related to mobility-related fall risk are vision, fast walking and cognitive functions. The literature point to no conclusions regarding the mobility safety benchmarks assessment in an integrated approach.

Conclusion: Our literature review shows a complete lack of a comprehensive well-tested clinical assessment tool for measuring mobility safety in stroke. The review propose future research using Dynamic Visual Acuity test as an integrated assessment methodology to assess mobility safety in chronic ambulatory stroke survivors.
\end{abstract}

Keywords: Stroke mobility, safety; Dynamic visual acuity.

\section{INTRODUCTION}

Following a stroke, independent ambulation is a major long-term goal after rehabilitation. Although $60 \%$ to $80 \%$ of stroke survivors achieve this goal. However, a typical course of physical therapy to increase walking speed does not guarantee that a person is "safe" in terms of their mobility. Therefore, a thorough understanding of performance benchmarks is critical to maximize safety and ensure quality performance. There is a debate over which types of performance benchmarks are the most useful for monitoring patients safety and remains a question of international concern especially after a stroke ${ }^{(1)}$. Presumably cognitive, visual and motor ability would have to be assessed ${ }^{(1)}$. The lack of such knowledge represents an important problem considering the high prevalence of mobility-related falls in this population. As $40 \%$ of all stroke survivors suffer serious falls within a year after their stroke, clinicians should be cautious when treating stroke patient to increase walking speed. The purpose of this literature review is to analyze and detail the available research what factors contribute to mobility falls in chronic ambulatory stroke survivors. Most importantly, the review proposes guidelines for an assessment battery combining the major threats assessed of mobility safety in integrated approach. We hope such effort will provide guidance for future scholars in the research area to enable the optimal recovery and maintenance of mobility safety especially after gait-oriented interventions in chronic ambulatory stroke survivors.

\section{MATERIAL AND METHODS}

In order to meet the objective of this narrative review, an electronic database search in PubMed/MEDLINE was performed to identify the available literature. This database was accessed online by one researcher through the local university's library system through January 2019 and was limited to articles written in English.

Specific key words and their combinations using the "AND" operator were used for the purpose of the literature search. These key words included: "Chronic stroke", "Gait," "Safety," "Mobility," "Fall risk".

Based on inclusion criteria, studies were accepted when: (1) they investigated the gait motor impairment/recovery and the mobility-related safety and falls (2) they included adult subjects who are clinically diagnosed with stroke (3) subjects were in 
the chronic stage of their injuries. We followed the PRISMA guidelines to depicts the flow of information through the phases of the narrative review.

The results from the selected literature were presented in four sections. We overviewed the literature about both the gait motor impairment (section one) and motor recovery (section two) following stroke and combined the gait impairment (as evidenced by decreased walking speed) with community ambulation (section three) to identify the gap between the gait disability and recovery in terms of mobility safety. Then, we discussed the literature regarding the major threats to mobility safety in chronic ambulatory stroke survivors (section four).
Finally, for the purpose of bridging the gap between gait disability and recovery and by identifying the interaction between the major threats to mobility safety, the discussion section proposed an assessment tool that will combine the three major factors in an integrated approach in chronic ambulatory stroke survivors.

\section{RESULTS}

Figure 1 is showing the publications identified and screened for eligibility in this narrative review. Following this screening process, thirty-three articles remained for further review for appropriateness and analysis.
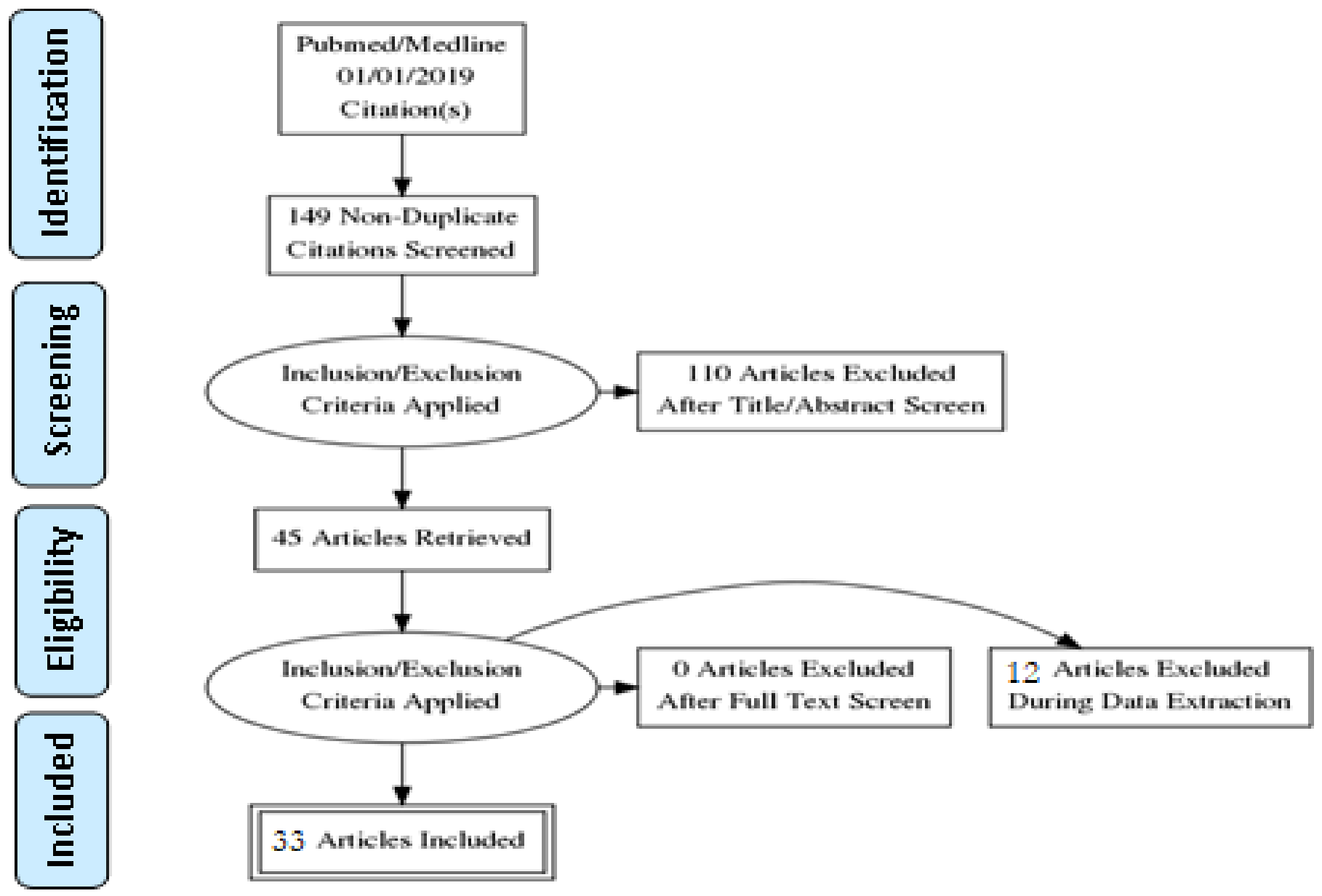

Figure 1: PRISMA flowchart of obtaining and eliminating articles functions 
The selected articles were grouped as the following:

(1) Gait motor impairment

(2) Gait motor recovery

(3) walking speed as it relates to the gait motor impairment and recovery post stroke (i.e. community ambulation)

(4) Threats to safe ambulation including fast walking, visual functions and cognitive

1) Gait motor Impairment: We found eight articles that addressed the post-stroke Gait motor Impairment ${ }^{(2-9)}$. Some sensory-motor deficits following a stroke may affect both sides of the body with more evident deficits in the contralateral to the lesioned brain hemisphere and include: weakness ${ }^{(2)}$, postural instability ${ }^{5}$ and abnormal movement synergies ${ }^{(6)}$. Subsequently, significant gait impairments including decreased walking speed with decreased cadence ${ }^{(3)}$ and incorrect timing of components within a movement pattern ${ }^{(7)}$ persist even years following stroke. Considering the high mobility recovery rates following stroke ${ }^{8}$, post-stroke walking speeds are significantly impaired ${ }^{(4,9)}$.

(2) Gait motor recovery: With regard to Gait motor recovery, five articles were identified ${ }^{(10-14)}$. Functional recovery of gait were quantified using clinical and functional measures. Various interventions have been proposed that target improving mobility of stroke survivors $(11,14)$. Based on the observation from the literature, the desired outcomes following specialized stroke rehabilitation basically depend on two key elements: Training intensity and task-specificity ${ }^{(12)}$.

Regardless of which intervention is used, the criteria for assessing and defining the true motor recovery in term of safe mobility have been ambiguous, considering the high incident of frequent falls in chronic stroke survivors especially during Activities of Daily Living (ADLs) ${ }^{(15)}$. Lamontagne and Fung ${ }^{(13)}$ examined the extent to which stroke subjects could increase their overground walking speed with respect to speed and unloading changes in different walking conditions. The study concluded that stroke subjects can increase substantially their walking speed without deleterious effects given proper instructions and a "safe" environment. In real-life, no guarantees on safe mobility is feasible as it is considered unpredictable (open) environment (16). Abreu (10) examined the effect of environmental predictability on postural control following stroke. They concluded that patient safety performance must be assessed as part of motor recovery in both predictable (closed) and unpredictable (open) environment taking into considerations all factors associated with mobility-related falls especially when considering walking speed and how it relate to recovery in terms of community ambulation.

(3) Walking speed and community ambulation: As for walking speed and how it relates to community ambulation, four articles were investigated (17-20).
Considering the critical role of walking speed for effective community ambulation, stroke has been shown to significantly contribute to long term disability and declined QOL and restricted community ambulation (20). Stroke survivors walk at speeds ranging from 0.4 to 0.85 $\mathrm{m} / \mathrm{s}$ (20), which may be insufficient for effective community ambulation. However. usual walking speed after a stroke can be ranked into clinically meaningful functional community ambulation classes, including household ambulation (less than $0.4 \mathrm{~m} / \mathrm{s}$ ), limited community ambulation ( 0.4 to $0.8 \mathrm{~m} / \mathrm{s})$, and full community ambulation (greater than $0.8 \mathrm{~m} / \mathrm{s}$ ) ${ }^{(18)}$. More specifically, A study by Van de Port et al. ${ }^{(20)}$ revealed a cutoff point of $0.66 \mathrm{~m} / \mathrm{s}$ as an indicator for unlimited (full) community ambulation in chronic stroke survivors. However, recent studies highlighted the need for research to inform a theoretical framework for measuring community ambulation in term of threats to safe ambulation, from which a battery of measurements can be developed and tested ${ }^{(17,19)}$.

\section{(4) Factors related to mobility-related falls:}

Three major threats to safe ambulation have been identified including fast walking, visual functions and cognitive functions.

(4.a) Fast walking: Five articles addressed fast walking as a major threat to safe ambulation (21-25). Walking in everyday life (i.e. community ambulation) necessitates walking adaptability based on time constraints, which is the ability to modify walking to meet behavioral demands of the environment such as crossing an intersection or rushing to the bathroom. Therefore, Individuals with limited ability to appropriately adjust their walking speed may either avoid walking fast as safety strategy or experience a heightened risk of falls when required to walk under such behavioral demands ${ }^{(26)}$. Stroke results in compromised ability to increase walking speed above the selected speed ${ }^{(21,24)}$. Walking speed is considered the most frequently reported activity (up to 90\%) at the time of a fall in stroke survivors ${ }^{(22,27)}$, suggesting walking speed is one of the major mobility threats to falls in ambulatory stroke survivors.

(4.b) Visual functions: Five articles addressed visual functions ${ }^{(28-32)}$. Intact visual functions are required and are significantly involved in spatial orientation especially during dynamic tasks such as walking ${ }^{(33)}$. A multi-centre prospective observation study was undertaken on 323 stroke survivors revealed that $92 \%$ of them had visual impairments ${ }^{(31)}$. Impaired vision has been shown to be largely related to poor QOL ${ }^{(28)}$ and mortality in older adults and it is reasonable to conclude that stroke survivors could share higher levels of thread ${ }^{(31)}$. The relationship between altered visual functions and mobility has been well addressed. Studies have shown that the preferred (self-selected) walking speed were largely dependent on visual functions, i.e. the better the visual functions the higher is the preferred walking speed ${ }^{(29)}$. In 
stroke survivors, the decreased walking speed, albeit pathological, may be a preferred walking speed and a behavioral compensation to maintain visual functions and a "safer" mechanism to maintain balance than walking at higher speeds ${ }^{(32)}$. Balance ${ }^{(30)}$ and vision ${ }^{(29)}$ are largely interrelated (31) and are significantly affected by increasing walking speed. All together, although increasing the walking speed in stroke patients is necessary for improving QOL and a major goal after rehabilitation, we found no evidence to whether it is "safe" to increase the walking speed of those patient without examining their Visual interaction with ambulation and how it would relate to their balance. So, it remains unclear what contributions gait training interventions would have to enable stroke patients to professionally master their mobility in order to maintain sufficient visual inputs and to reduce risk of fall especially when various challenges are being added that could pose a threat on their safety during locomotion. (4.c) Cognitive functions: six articles addressed cognitive functions ${ }^{(34-39)}$. Walking at self-selected speed is an automated rhythmic motor behavior that can be performed without the need of high level of cognitive functions, but still requires an intact cognition (40). Cognitive dysfunctions are common complications after stroke with prevalence ranging from $20 \%$ to $80 \%{ }^{(34)}$. Studies that has been done on stoke patients have shown significant effect of these cognitive challenges on mobility and balance ${ }^{(35-38)}$. A study has shown that even full ambulatory stroke survivors with intact cognitive function have difficulties performing two tasks, such as performing an over-ground talking-while-walking task (39). Consequently, it is reasonable to conclude the strong association between impaired cognitive function and balance as major threats associated with falls and ADLs performance ${ }^{(41)}$.

\section{DISCUSSION}

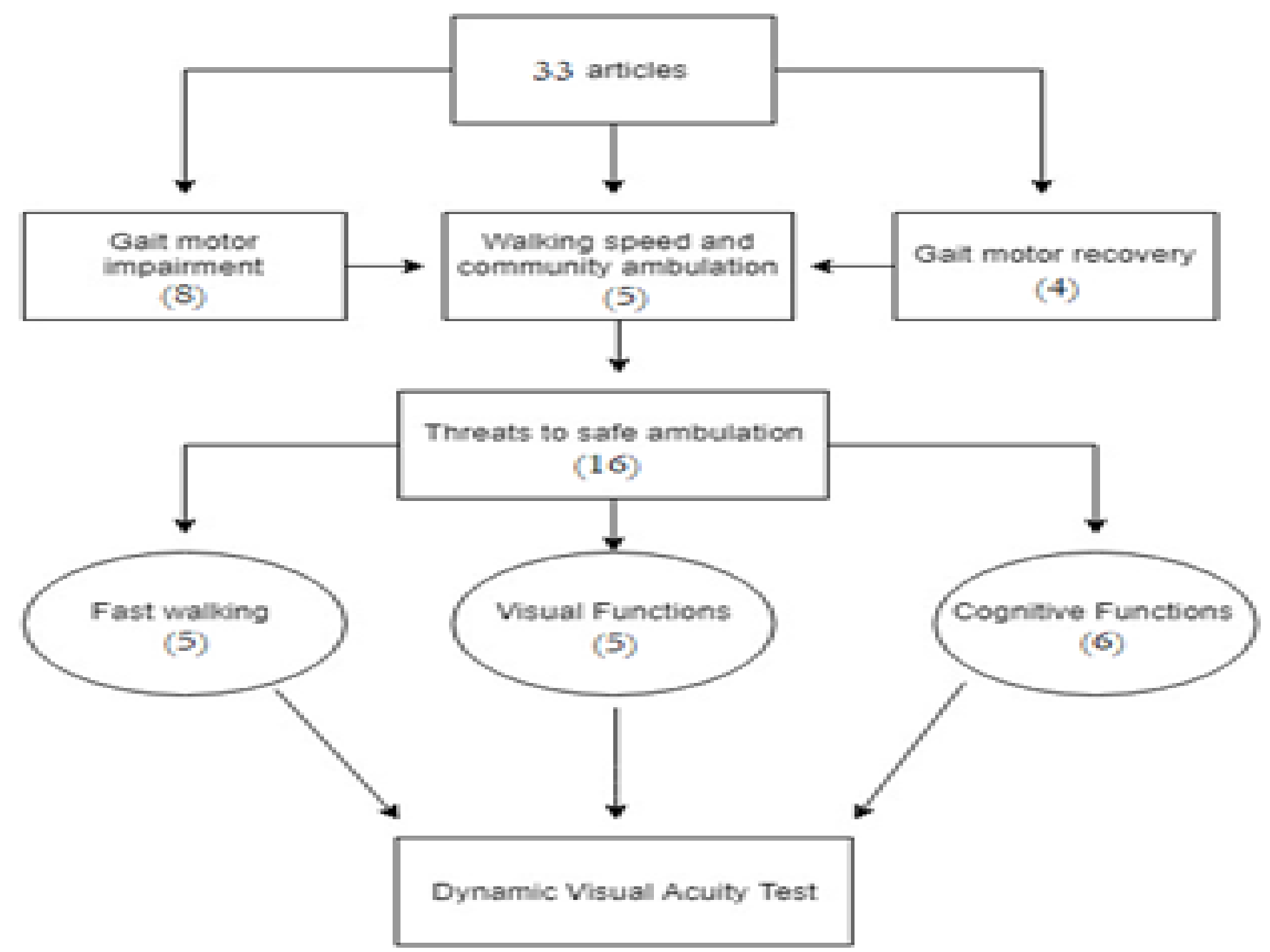

Figure 2. Flow diagram showing the narrative review process from results through our discussion. 
As independent and fast ambulation is a major long-term goal after rehabilitation, our results from the literature can point to no conclusions regarding the walking safety at high speeds following gaitoriented training compared to pre-training condition. During the recovery process, especially following rehabilitation, one can reasonably argue that the true motor recovery of gait following stroke has not been fully addressed.

Figure 2: Flow diagram of the narrative review process

First, researchers have suggested that given the appropriate training paradigm, motor improvement in gait can be achieved in the chronic stage of stroke. Though, in the chronic stage, stroke patients are at higher risk of falls during mobility (23). Second, factors related to fall in chronic stage are not entirely examined following rehabilitation and after discharge. For example, stroke patients have limited capacity to increase their walking speed and their community ambulation is determined by reactive balance, i.e. how they react with the environment to maintain balance during locomotion ${ }^{(20)}$. Accordingly, stroke patients in everyday life may experience different neuro-muscular and cognitive challenges or even both. So we still don't know what contributions do cognitive functions have on the outcomes and subsequently how this would affect balance especially with impaired visual functions and/or during unpredictable increasing mobility demands as when increasing walking speed.

Third, researchers have indicated that clinicians should be cautious when treating stroke patient to increase their walking speed, and that there is a need for assessment batteries that assess reactive balance and vision especially during environmental interaction that would require those patients to walkand-talk (35) or walk faster ${ }^{(23)}$ along with their common visual deficits ${ }^{(31)}$. However, there is a lack of understanding for the safety associated with the behavioral changes (mobility) of stroke survivors as part of the motor impairments especially under conditions that are commonly experienced and could negatively affect their mobility and stability. This understanding will be necessary to overcome the motor impairments following stroke and help researchers and clinicians understand the potential contribution of cognitive and visual functions to the patients' capabilities to interact with various distractions in their communities. Thus, there is an urgent need for an assessment battery to assess mobility safety pre-and-post gait training interventions. Before initiating an exercise intervention to increase walking speed, threats to safe ambulation must be assessed not only separately, but also comprehensively.

Three threats to mobility safety can be identified from the literature: Limited capacity to walk fast, cognitive deficits and visual impairments. In light of this, dynamic visual acuity (DVA) is defined as the ability to maintain visual acuity (i.e. clear vision) during head movements associated with dynamic tasks such as walking, so that more negative score indicates better vision ${ }^{(42)}$. DVA is subserved by the vestibular system through vestibulo-ocular reflex (VOR). VOR generates compensatory eye movement in the opposite direction of head movement to stabilize images on the fovea to maintain visual acuity. In addition to compensatory eye movement, it has been also shown that during locomotion, the fullbody segmental kinematics should be modified to reduce perturbations to the head ${ }^{(43)}$, suggesting that in addition to VOR, the body may have other mechanisms that contribute to improving gaze control and maintaining DVA ${ }^{(42-44)}$. Moreover, less steady gait, as in dual-task walking ${ }^{(37)}$ or fast walking, will increase perturbation to the head and negatively affect DVA. Aging studies have shown that the agerelated decrease in VOR and walking speed may not be pathological but an adaptive response to increase stability and maintain balance and intact visual inputs by decreasing perturbations from the body segments to the head associated with increasing walking speed (45), which further support that changes in body kinematics may influence DVA. This information is important for two reasons. First, clear vision is affected by walking speed, unsteady gait and declined postural control ${ }^{(5)}$, which all deteriorate following stroke $(5,31,45)$. Second, older patient with and without stroke are highly dependent on the clarity of the visual information for their overall postural control $^{(5,33)}$ and overall locomotion ${ }^{(29)}$ so that blurred vision during dynamic tasks can significantly jeopardize their stability. However, DVA has never been assessed as a safety outcome following gait training rehabilitation to address visual changes along with the changes in walking abilities.

The proposed DVA test was first described in 2005 as a diagnostic tool to assess the ability to visually resolve targets during treadmill walking at one fixed speed and on healthy participants ${ }^{(42)}$. The significance of this testing methodology was that it allowed the researchers to understand visual acuity changes during dynamic activities of daily living such as walking. 
In fact, considering the relationship between walking speed, cognition, balance, quality of walking and DVA, the final DVA score could be an indicator of participants' ability to interact with various challenges that would negatively affect their balance and the quality of walking. Specifically, the participants will be talking (naming the direction of the "C" optotypes while (2) walking on a treadmill at a specific or different speeds and performing a dynamic visual acuity test presented on a computer screen at eye level ${ }^{(42)}$. Therefore, DVA testing addresses the cognitive, fast-walking and visual challenges, respectively, which are the three major threats to mobility safety identified from the literature. In other words, a significant improvement in DVA and balance scores following an intervention would indicate that the participants were able to not only walk fast but also to master their mobility more efficiently. Therefore, this analysis suggests future research on DVA as a possible integrated approach to answer the question: is it actually safe for chronic ambulatory stroke survivors to walk faster? It is our hope that the results from the review will guide future researched on such important aspect of rehabilitation which will increase our understanding for the expected motor recovery following different gait interventions and raise the hope for developing motor disability following stroke.

In conclusion, the purpose of this literature review is to highlight the need for an evidence-based assessment battery to assess mobility safety in chronic ambulatory stroke survivors. The current research demonstrates that gait-oriented training can enhance mobility capacity of this population. However, physical therapy interventions targeting enhance mobility capacity such as increasing walking speed does not guarantee that a person is safe. This research highlights DVA for further research as an assessment methodology that combines three factors that are related to mobility-related fall risk, i.e. vision, fast walking and dual-task walking. It is our goal to introduce an assessment battery that researchers and clinicians can possibly adopt to assess the safety of their patients before sending them to their communities with their sensorimotor pathologies and more importantly in geriatric stroke population. It also should be interpreted as a need for integrating other gait intervention programs that focuses on improving the cognitive functions and balance taking into consideration their effect on visual functions of stroke survivors during walking.

\section{REFERENCES}

1.Xu T, Clemson L, O'Loughlin K, Lannin NA, Dean C, Koh G (2018): Risk factors for falls in community stroke survivors: A systematic review and meta-analysis. Archives of Physical Medicine and Rehabilitation, 99:563573.e565.

2.Bourbonnais D, Vanden Noven $S$ (1989): Weakness in patients with hemiparesis. Am J Occup Ther., 43:313-319.

3.Brandstater ME, de Bruin H, Gowland C, Clark BM (1983): Hemiplegic gait: Analysis of temporal variables. Arch Phys Med Rehabil., 64:583-587.

4.Hill K, Ellis P, Bernhardt J, Maggs P, Hull S (1997): Balance and mobility outcomes for stroke patients: A comprehensive audit. Aust J Physiother., 43:173-180.

5.Peurala SH, Kononen P, Pitkanen K, Sivenius J, Tarkka IM (2007): Postural instability in patients with chronic stroke. Restor Neurol Neurosci., 25:101-108.

6.Shiavi R, Bugle HJ, Limbird T (1987): Electromyographic gait assessment, part 2: Preliminary assessment of hemiparetic synergy patterns. J Rehabil Res Dev., 24:2430.

7.von Schroeder HP, Coutts RD, Lyden PD, Billings E, Jr., Nickel VL (1995): Gait parameters following stroke: A practical assessment. J Rehabil Res Dev., 32:25-31.

8.Baer G, Smith M (2001): The recovery of walking ability and subclassification of stroke. Physiother Res Int., 6:135144.

9.Eng JJ, Chu KS, Dawson AS, Kim CM, Hepburn KE (2002): Functional walk tests in individuals with stroke: Relation to perceived exertion and myocardial exertion. Stroke, 33:756-761.

10.Abreu BC (1995): The effect of environmental regulations on postural control after stroke. Am J Occup Ther., 49:517525.

11.Belda-Lois JM, Mena-del Horno S, Bermejo-Bosch I, Moreno JC, Pons JL, Farina D et al. (2011): Rehabilitation of gait after stroke: A review towards a topdown approach. J Neuroeng Rehabil., 8:66.

12.Foley NC, Teasell RW, Bhogal SK, Speechley MR (2003): Stroke rehabilitation evidence-based review: Methodology. Top Stroke Rehabil., 10:1-7.

13.Lamontagne A, Fung J (2004): Faster is better implications for speed-intensive gait training after stroke. Stroke, 35:2543-2548.

14.van de Port IG, Wood-Dauphinee S, Lindeman E, Kwakkel G (2007): Effects of exercise training programs on walking competency after stroke: A systematic review. Am J Phys Med Rehabil., 86:935-951.

15.Cho K, Yu J, Rhee H (2015): Risk factors related to falling in stroke patients: A cross-sectional study. J Phys Ther Sci., 27:1751-1753.

16.Shumway-Cook A Woollacott MH (2012): Motor control: Translating research into clinical practice. Wolters Kluwer, Lippincott Williams \& Wilkins.

17.Lord SE, Rochester L (2005): Measurement of community ambulation after stroke. Stroke, 36:1457-1461. 
18.Schmid A, Duncan PW, Studenski S, Lai SM, Richards L, Perera S et al. (2007): Improvements in speed-based gait classifications are meaningful. Stroke, 38:2096-2100.

19.Wesselhoff S, Hanke TA, Evans CC (2018): Community mobility after stroke: A systematic review. Topics in Stroke Rehabilitation, 25:224-238.

20.van de Port IG, Kwakkel G, Lindeman E (2008): Community ambulation in patients with chronic stroke: How is it related to gait speed? J Rehabil Med., 40:23-27.

21.Balasubramanian CK, Clark DJ, Fox EJ (2014): Walking adaptability after a stroke and its assessment in clinical settings. Stroke Res Treat., 2014:591013.

22.Forster A, Young J(1955): Incidence and consequences of falls due to stroke: A systematic inquiry. BMJ: British Medical Journal,311:83-86

23.Harris JE, Eng JJ, Marigold DS, Tokuno CD, Louis CL (2005): Relationship of balance and mobility to fall incidence in people with chronic stroke. Phys Ther., 85:150-158.

24.Robinson CA, Shumway-Cook A, Matsuda PN, Ciol MA (2011): Understanding physical factors associated with participation in community ambulation following stroke. Disabil Rehabil., 33:1033-1042.

25.Tyrell CM, Roos MA, Rudolph KS, Reisman DS (2011): Influence of systematic increases in treadmill walking speed on gait kinematics after stroke. Phys Ther., 91:392403.

26.Patla AE, Shumway-Cook A (1999): Dimensions of mobility: Defining the complexity and difficulty associated with community mobility. Journal of Aging and Physical Activity, 7:7-19.

27.Jørgensen L, Engstad T, Jacobsen BK (2002): Higher incidence of falls in long-term stroke survivors than in population controls. Stroke, 33:542-547.

28.Langelaan M, de Boer MR, van Nispen RM, Wouters B, Moll AC, van Rens GH (2007): Impact of visual impairment on quality of life: A comparison with quality of life in the general population and with other chronic conditions. Ophthalmic Epidemiol., 14:119-126.

29.Leat SJ, Lovie-Kitchin JE (2008): Visual function, visual attention, and mobility performance in low vision. Optom Vis Sci., 85:1049-1056.

30.Orendurff MS, Segal AD, Klute GK, Berge JS, Rohr ES, Kadel NJ (2004): The effect of walking speed on center of mass displacement. J Rehabil Res Dev., 41:829834.

31.Rowe F, Brand D, Jackson CA, Price A, Walker L, Harrison S et al. (2009): Visual impairment following stroke: Do stroke patients require vision assessment? Age Ageing, 38:188-193.

32.Titianova EB, Peurala SH, Pitkanen K, Tarkka IM (2008): Gait reveals bilateral adaptation of motor control in patients with chronic unilateral stroke. Aging Clin Exp Res., 20:131-138.

33.Chokron S, Colliot P, Bartolomeo P (2004): The role of vision in spatial representation. Cortex, 40:281-290.

34.Sun JH, Tan L, Yu JT (2014): Post-stroke cognitive impairment: Epidemiology, mechanisms and management. Ann Transl Med., 2:80.

35.Plummer-D'Amato P, Altmann LJ, Saracino D, Fox E, Behrman AL, Marsiske M (2008): Interactions between cognitive tasks and gait after stroke: A dual task study. Gait Posture, 27:683-688.

36.Hyndman D, Ashburn A, Yardley L, Stack E (2006): Interference between balance, gait and cognitive task performance among people with stroke living in the community. Disabil Rehabil., 28:849-856.

37.Yang YR, Chen YC, Lee CS, Cheng SJ, Wang RY (2007): Dual-task-related gait changes in individuals with stroke. Gait Posture, 25:185-190.

38.Haggard P, Cockburn J, Cock J, Fordham C, Wade D (2000): Interference between gait and cognitive tasks in a rehabilitating neurological population. J Neurol Neurosurg Psychiatry, 69:479-486.

39.Bowen A, Wenman R, Mickelborough J, Foster J, Hill E, Tallis R (2001): Dual-task effects of talking while walking on velocity and balance following a stroke. Age Ageing, 30:319-323

40.Yogev-Seligmann G, Hausdorff JM, Giladi N (2008): The role of executive function and attention in gait. Mov Disord., 23:329-342; quiz 472.

41.Cho K, Yu J, Rhee H (2015): Risk factors related to falling in stroke patients: A cross-sectional study. Journal of physical therapy science, 27:1751-1753.

42.Peters BT, Bloomberg JJ (2005): Dynamic visual acuity using "far" and "near" targets. Acta Otolaryngol., 125:353357

43.Mulavara AP, Bloomberg JJ (2002): Identifying headtrunk and lower limb contributions to gaze stabilization during locomotion. J Vestib Res. 12:255-269.

44.Imai T, Moore ST, Raphan T, Cohen B (2001): Interaction of the body, head, and eyes during walking and turning. Exp Brain Res., 136:1-18.

45.Judge JO, King MB, Whipple R, Clive J, Wolfson LI (1995): Dynamic balance in older persons: Effects of reduced visual and proprioceptive input. J Gerontol A Biol Sci Med Sci., 50:M263-270. 